\section{Dissociation of broadband high-frequency activity and neuronal firing in the neocortex}

\author{
Marcin Leszczyński ${ }^{1,2 *}$, Annamaria Barczak² ${ }^{2}$ Yoshinao Kajikawa ${ }^{2}$, Istvan Ulbert ${ }^{3}$, \\ Arnaud Y. Falchier ${ }^{2,4}$, Idan Tal ${ }^{1,2}$, Saskia Haegens ${ }^{1,5}$, Lucia Melloni ${ }^{6}$, \\ Robert T. Knight ${ }^{7}$, Charles E. Schroeder ${ }^{1,2 *}$
}

\begin{abstract}
Broadband high-frequency activity (BHA; 70 to $150 \mathrm{~Hz}$ ), also known as "high gamma," a key analytic signal in human intracranial (electrocorticographic) recordings, is often assumed to reflect local neural firing [multiunit activity (MUA)]. As the precise physiological substrates of BHA are unknown, this assumption remains controversial. Our analysis of laminar multielectrode data from V1 and A1 in monkeys outlines two components of stimulus-evoked BHA distributed across the cortical layers: an "early-deep" and "late-superficial" response. Early-deep BHA has a clear spatial and temporal overlap with MUA. Late-superficial BHA was more prominent and accounted for more of the BHA signal measured near the cortical pial surface. However, its association with local MUA is weak and often undetectable, consistent with the view that it reflects dendritic processes separable from local neuronal firing.
\end{abstract}

\section{INTRODUCTION}

Broadband high-frequency activity (BHA; 70 to $150 \mathrm{~Hz}$ ), also known as "high gamma," a key analytic signal in human intracranial recordings, is often assumed to reflect local neural firing [multiunit activity (MUA)]. Accordingly, BHA has been used to study neuronal population responses reflecting auditory $(1,2)$, visual $(3-5)$, language (6), mnemonic (7-10), and cognitive control $(11,12)$. BHA is arguably the electrophysiological measure best correlated with the blood oxygenation level-dependent (BOLD) signal in functional magnetic resonance imaging (fMRI) $(13,14)$. However, beyond the fact that BHA correlates with neuronal spiking $(13,15-17)$, its precise physiology remains unknown; specifically, the neuronal populations and physiological processes generating BHA have not been identified. Here, we show that BHA can be dissociated from MUA in the primary visual and auditory cortex. Analyzing laminar multielectrode data recorded in monkeys, we found a bimodal distribution of stimulus-evoked BHA across the layers of cortex: an "early-deep" and "late-superficial" response. The early-deep BHA had a clear spatial (laminar) and temporal association with local MUA; however, the spatiotemporal overlap of MUA with the late-superficial BHA signal was much less clear. In many cases, particularly in V1 (70\%), supragranular sites showed a strong BHA in lieu of any detectable increase in MUA. Because of volume conduction, BHAs from both the earlydeep and the late-superficial generators contribute to the field potential (FP) at the pial surface, but the contribution is weighted toward the superficial BHA. Although both BHA components volume conduct to the pial surface, the strongest generators of BHA are in the

\footnotetext{
${ }^{1}$ Cognitive Science and Neuromodulation Program, Departments of Psychiatry, Neurology and Neurosurgery, Columbia University College of Physicians and Surgeons, New York, NY, USA. ${ }^{2}$ Translational Neuroscience Division of the Center for Biomedical Imaging and Neuromodulation, Nathan Kline Institute, Orangeburg, NY, USA. ${ }^{3}$ Institute for Cognitive Neuroscience and Psychology, Research Centre for Natural Sciences, Hungarian Academy of Sciences, Budapest, Hungary. ${ }^{4}$ Department of Psychiatry, NYU Grossman School of Medicine, New York, NY, USA. 'Donders Institute for Brain, Cognition and Behaviour, Radboud University Nijmegen, Nijmegen, Netherlands. ${ }^{6}$ Department of Neurology, New York University Langone Health, New York, NY, USA. ${ }^{7}$ Department of Psychology and Helen Wills Neuroscience Institute, University of California at Berkeley, Berkeley, CA, USA.

*Corresponding author. Email: leszczynski.marcin@gmail.com (M.L.); cs2388@ columbia.edu (C.E.S.)
}

superficial cortical layers. The origins of BHA include a mixture of the neuronal action potential firing and dendritic processes separable from firing. It is likely that, as typically recorded, BHA signals emphasize the latter processes to a greater extent than previously recognized.

\section{RESULTS}

\section{Two BHA components: Early deep and late superficial}

We investigated the neural mechanisms generating BHA signal and their relationship to MUA in the neocortex by analyzing FP and concomitant MUA signals recorded with laminar multielectrodes in macaque primary visual (V1: two animals, 104 experimental sessions with whole-screen flashes, 49 sessions with free viewing) and auditory (A1: two animals, 26 experimental sessions with broadband noise, 26 sessions with best frequency tone) cortices. We used first and second derivative (current source density or "CSD") analyses of the laminar FP profiles, along with a concomitant MUA to localize the neuronal generators of BHA and to determine their spatiotemporal relationship to neuronal firing. Figure 1 displays representative laminar activity profiles recorded from primary visual (Fig. 1, A, B, and E) and auditory (Fig. 1, C, D, and F) cortices. The notion that BHA directly reflects neuronal firing raises the obvious prediction that $\mathrm{BHA}$ and MUA should have the same spatial and temporal distributions across cortical layers. Contrary to this prediction, however, the spatiotemporal profiles of BHA and MUA were reliably different. We observed two temporally and spatially distinct BHA components, including the early-deep BHA localized to the granular and infragranular layers and the late-superficial BHA observed in the supragranular layers (Fig. 1, E and F). Early-deep BHA had clear spatiotemporal overlap with granular and infragranular MUA. However, the spatiotemporal association between late-superficial BHA and MUA was much less clear. Late-superficial BHA often had little or no MUA concomitant, particularly in $\mathrm{V} 1$.

Consistent with earlier work $(13,15-17)$, we found that MUA and BHA were correlated at all cortical depths in both V1 and A1 (all Spearman's rho, $>0.76$; all $P<0.01$ ). We used cross-correlation between BHA and MUA at supragranular, granular, and infragranular layers to test whether the relation between these two signals changes across cortical depth. The cross-correlation coefficient profiles were 


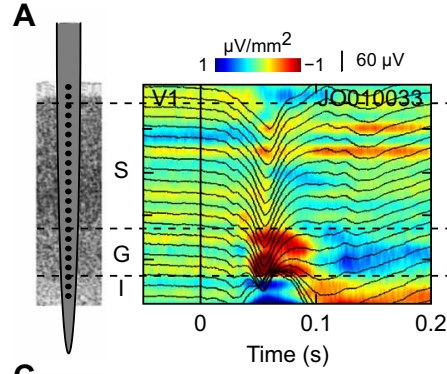

C

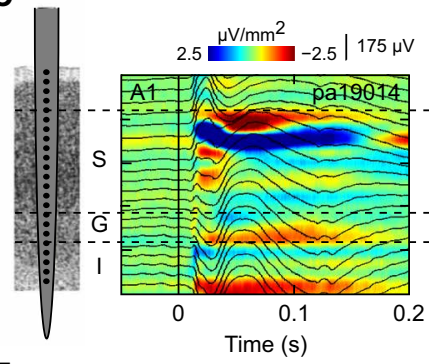

E

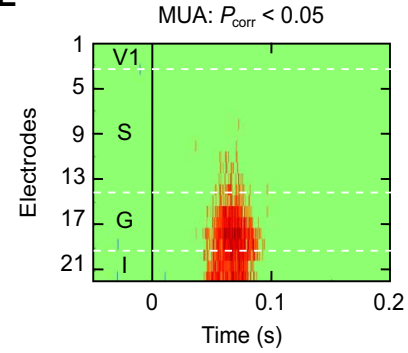

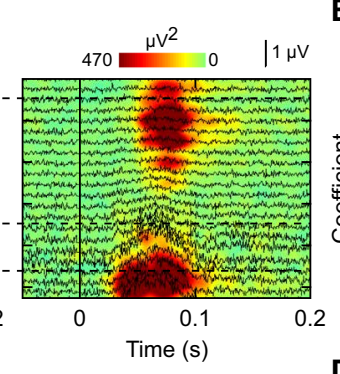

B
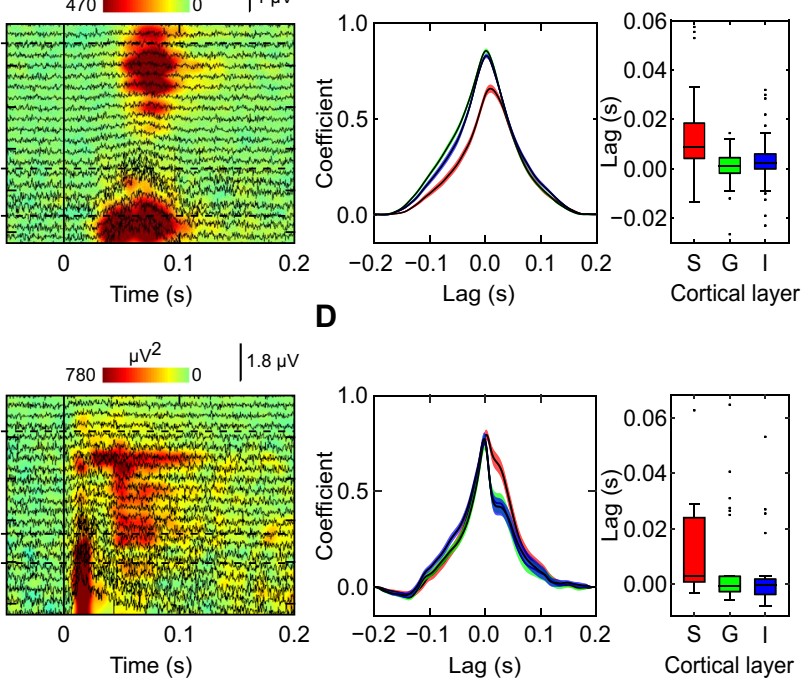

BHA: $P_{\text {corr }}<0.05$
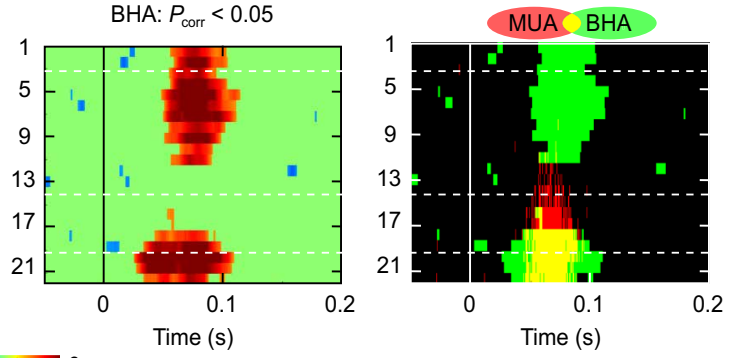

$\mathbf{F}$
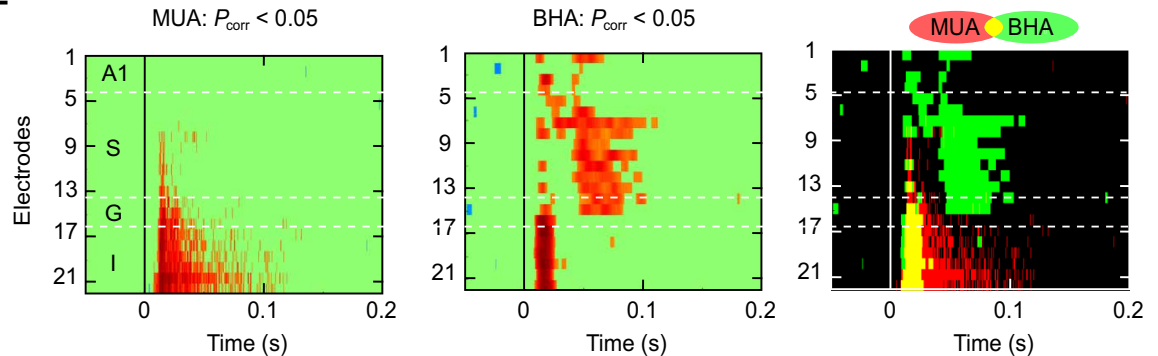

Fig. 1. Laminar activity profiles from individual penetrations in V1 and A1. Color maps show a CSD superimposed with FPs (left) and a BHA (color map) superimposed with MUA (line plots) profiles (right) in V1 (A) and A1 (C) from a representative session. (A) presents data from V1 recordings during diffuse flash stimulation. (C) shows data from $A 1$ recordings during presentation of broadband noise (100-ms duration). $x$ axes indicate time relative to stimulus onset. $y$ axes represent cortical depth with increasing numbers from superficial to deep layers. Vertical lines indicate sensory events. (B and $\mathbf{D}$ ) Line plots show the distribution of MUA-BHA cross-correlation coefficients calculated across trials for each penetration of V1 (B) and A1 (D). Box plot presents distribution of the lags obtained from the cross-correlations. Positive values indicate that MUA leads BHA. Shading in the line plot reflects SEM. Central mark and edges in box plots show median and 25 th and 75 th percentiles. (E and F) show maps of $z$ statistics calculated across trials $(n=137$ and 84$)$ for individual V1 $(E)$ and A1 (F) penetration. The statistics values are obtained from a nonparametric pairwise test on MUA (left) and BHA (middle) relative to the prestimulus baseline. Nonsignificant time points are masked $(P<0.05$; controlled for multiple comparisons across channels and time with the Benjamini and Yekutieli procedure). Right panels show an overlap (yellow) between the BHA (green) and MUA (red) statistic masks.

nonuniform across layers (Fig. 1, B and D). Specifically, the coefficients in the supragranular layers peaked later, relative to those in deeper layers [median lags in V1 supragranular, granular, and infragranular layers were 9, 1, and $2.5 \mathrm{~ms}$; while median lags in A1 supragranular, granular, and infragranular layers were $3.25,-0.5$, and $-0.25 \mathrm{~ms}$; both Kruskal-Wallis (KW) tests, $P<0.01$; Fig. 1 , B and D]. This suggests that the relationship between BHA and MUA might vary rather than being constant across cortical depth. To understand this divergence, we quantified the laminar/temporal distributions of both signals, which confirmed the above impressions about the changing association between BHA and MUA across cortical depth.

\section{Distinct laminar and temporal distributions of early-deep and late-superficial BHA}

We tested laminar distributions of BHA and MUA across four different experiments in two cortical areas, V1 and A1. First, we used diffused light flash stimulation (V1-DF), which weakly activates the supragranular layers of V1 relative to patterned stimulation (18); 
this contrast has been known for decades [see, e.g., (19)]. We selected these nonoptimal stimuli because we expected the dissociation to be strongest under these conditions, where activation of the superficial layers is relatively weak. We observed that BHA and MUA distributions were both nonuniform across V1 cortical depth (both KW tests, $P<0.001, n=104$ ), yet they differed markedly (Fig. 2, A to D). BHA was strongest in layers where neural firing was sparse to undetected. It was more pronounced in the supragranular than both granular and infragranular layers (all Wilcoxon tests; $P<0.001$ ). MUA displayed the opposite pattern: It was decreased in the supragranular compared to both granular and infragranular layers (both Wilcoxon tests; $P<0.001)$. BHA magnitude $(P=0.03)$ unlike MUA $(P=0.14)$ did differ across the granular and infragranular layers. Seventy percent of all V1 penetrations with diffused light flash stimulation showed a strong supragranular BHA in lieu of any detectable MUA increase. The fact that the BHA and MUA have a differing laminar/temporal distributions accords with the view that these signals reflect different aspects of neural activity.

To test whether the observed dissociation generalizes across types of visual stimuli and task, we examined BHA-MUA dissociation in experimental data collected during free viewing of natural scenes in the same V1 penetrations (V1-FV; see Materials and
Methods). It is noteworthy that natural scene viewing produces a much more effective activation of the superficial layers of the primary visual cortex (20). There are multiple differences in BHA and MUA morphology elicited by "active" (i.e., fixation locked) as compared to "passive" (stimulus locked) visual input. For example, both BHA and MUA decrease during saccade and rebound after saccade termination (Fig. 2, E to H). Despite these differences in both BHA and MUA morphology, we continue to observe the laminar dissociation between BHA and MUA. Both BHA and MUA show nonuniform distributions across layers (both KW tests, $P<0.001, n=49$ ), with superficial layers showing strongest BHA (all Wilcoxon tests, $z>5.7$, $P<0.001$ ) and weakest MUA (all Wilcoxon test, $z>6.0, P<0.001$ ).

Next, we sampled from A1 during presentation of broadband noise (A1-BBN; see Materials and Methods) to test whether these effects generalize to other areas of the sensory cortex. Despite differing temporal patterns of BHA signals in V1 and A1, key aspects of laminar dissociation between BHA and MUA generalize to A1. As in V1, event-related BHA and MUA were both observed across all three laminar compartments (Fig. 3, A to D), and both had a nonuniform laminar distribution (both KW tests, $P<0.001, n=26$ ). Critically, BHA was stronger in the supragranular layers compared to granular and infragranular layers (both Wilcoxon tests, $P<0.001$ ).
A

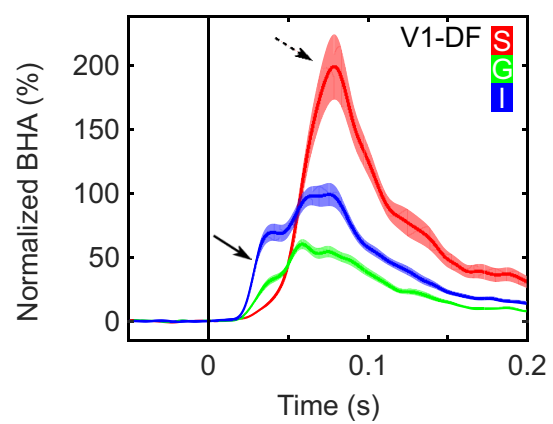

E

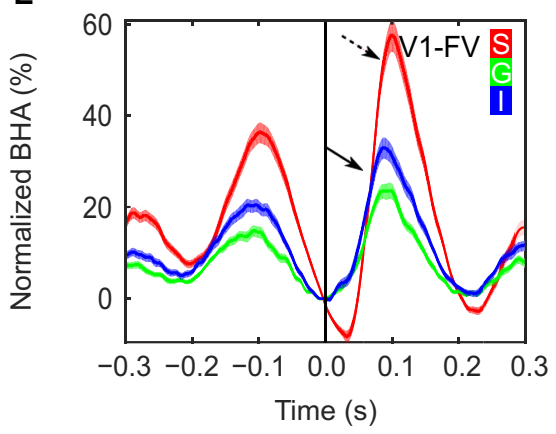

B

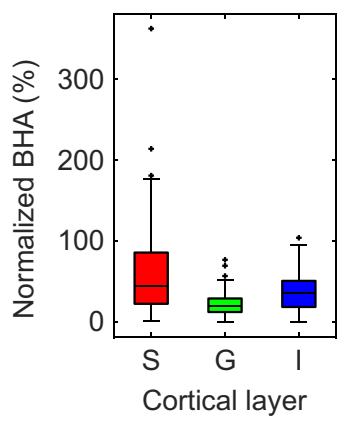

$\mathbf{F}$

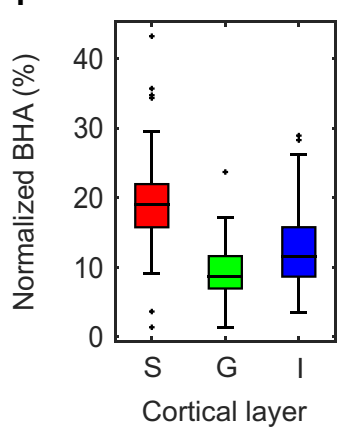

C

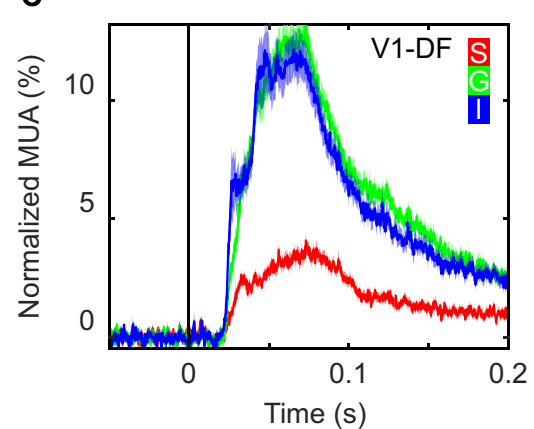

$\mathbf{G}$

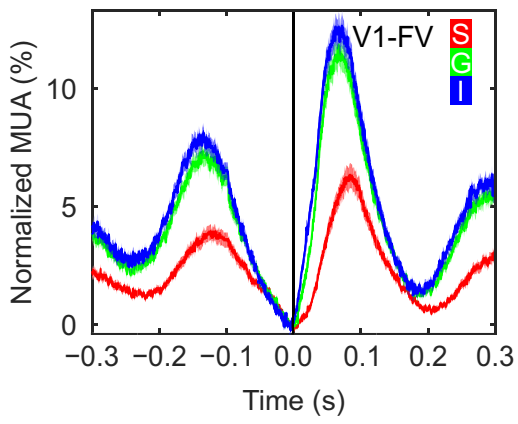

D

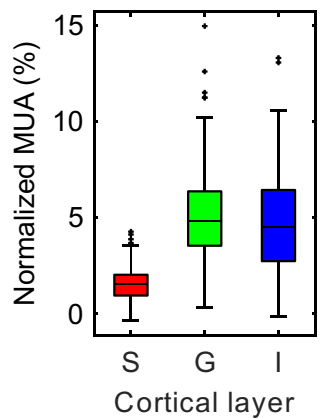

H

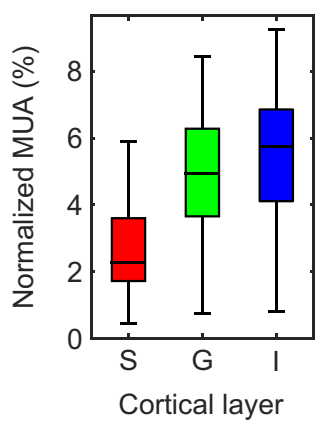

Fig. 2. Differential laminar distribution of BHA and MUA across experiments in V1. (A to D) present V1 data from recordings during diffuse flash stimulation (V1-DF; $n=104$ experiments in two animals). (E to H) Data from V1 recordings during free-viewing exploration of visual images [V1-FV; $n=49$, same two animals as in (A to D)]. Line plots show the time course of BHA ( $A$ and $E$ ) and MUA ( $C$ and $G$ ) response across supragranular, granular, and infragranular (red, green, and blue lines) layers. $x$ axes indicate time relative to stimulus $(A, C)$ or fixation onset $(E, G)$. $y$ axes represent signal change from baseline (i.e., normalized $B H A / M U A)$. Box plots present $B H A$ ( $B$ and $F$ ) and MUA ( $D$ and $H$ ) distributions averaged across time after stimulus onset ( $B$ and $D)$ and across the entire pre- and postfixation epoch ( $F$ and $H)$. Supragranular, granular, and infragranular (S, G, and I) layers are plotted as separate box plots. Box plots indicate 25th percentile, median, and 75th percentile; whiskers extend to extreme values not considered outliers, while outliers are marked with crosses. Shading in line plots reflects SEM. Note the consistently different laminar distributions of BHA and MUA in both experiments. Despite different stimuli, levels of firing, and BHA magnitude across experiments, BHA in the supragranular layers is enhanced relative to that in the granular and infragranular layers, while MUA in supragranular is sparse compared to granular and infragranular. Note also that in the free viewing (E to $H)$, there is substantial saccadic modulations of MUA and BHA across all layers, with a clear pattern of MUA suppression around the time of the saccade (perisaccadic suppression) and MUA increase at the end of the saccade (onset of fixation). Arrows in (A and E) indicate early (solid line) and late (dashed line) BHA components. 
A

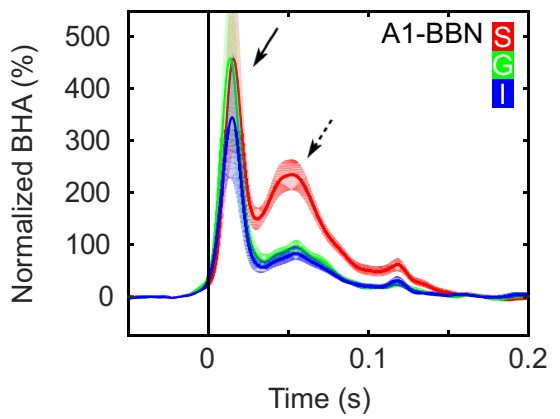

E

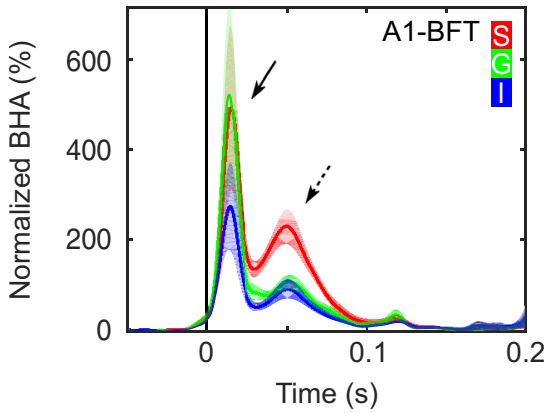

B

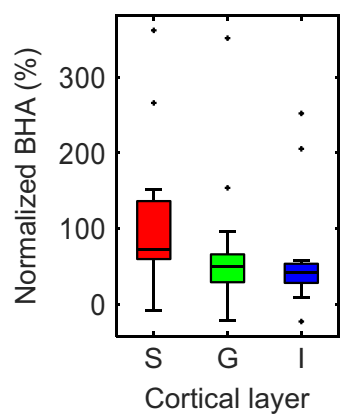

$\mathbf{F}$

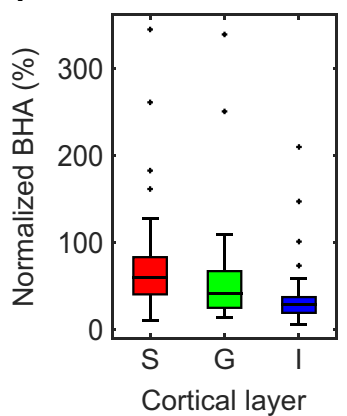

C

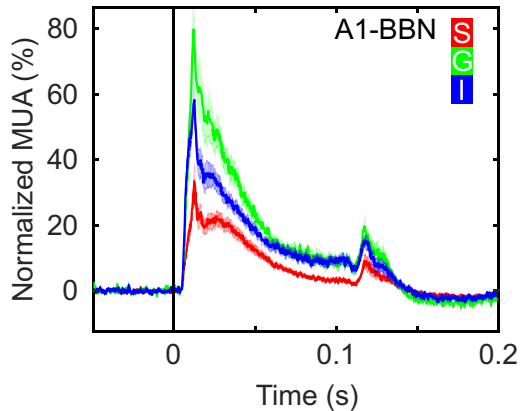

G

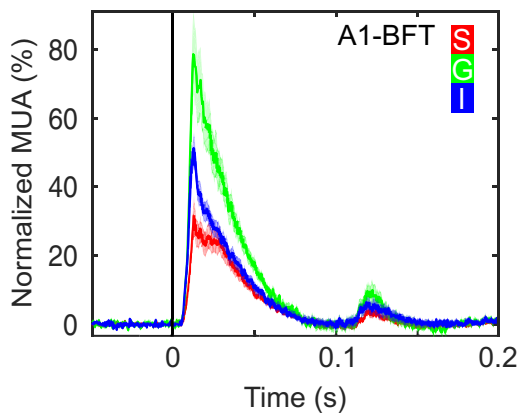

D

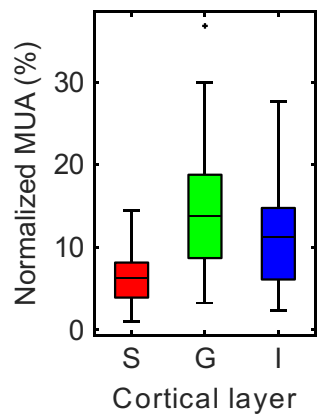

H

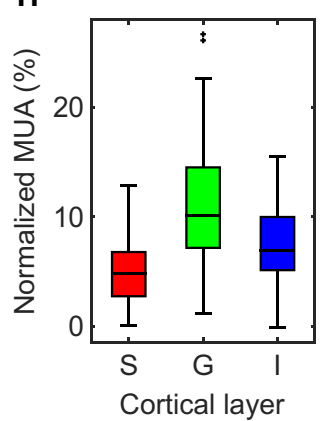

Fig. 3. Differential laminar distribution of BHA and MUA across experiments in $\mathbf{A} 1$. (A to $\mathbf{D})$ presents data from $A 1$ recordings during presentation of broadband noise (A1-BBN; duration, $100 \mathrm{~ms} ; n=26$, two animals). ( $E$ to $\mathbf{H}$ ) shows data from A1 recordings during presentation of best frequency tones [A1-BFT; 100-ms duration; $n=26$, same two animals as in (A to D)]. Line plots show the time course of BHA (A and $E$ ) and MUA (C and $G$ ) response across supragranular, granular, and infragranular (red, green, and blue lines) layers. $x$ axes indicate time relative to stimulus ( $A, C, E, G)$. $y$ axes represent signal change from baseline (i.e., normalized BHA/MUA). Box plots present BHA ( $\mathrm{B}$ and F) and MUA ( $\mathrm{D}$ and $\mathrm{H}$ ) distributions averaged across time after stimulus onset. Supragranular, granular, and infragranular ( $\mathrm{S}, \mathrm{G}$, and $\mathrm{I}$ ) layers are plotted as separate box plots. Box plots indicate 25th percentile, median, and 75th percentile; whiskers extend to extreme values not considered outliers, while outliers are marked with crosses. Shading in line plots reflects SEM. Note the consistently different laminar distributions of BHA and MUA in both experiments. Despite different stimuli (i.e., broadband noise and best frequency tones), BHA in the supragranular layers is enhanced relative to that in the granular and infragranular layers, while MUA in supragranular is sparse compared to granular and infragranular. Arrows in ( $\mathrm{A}$ and $\mathrm{E}$ ) indicate early (solid line) and late (dashed line) BHA components.

In contrast, supragranular MUA was weaker than granular and infragranular layers MUA (both Wilcoxon tests, $P<0.001$ ). The poststimulus time interval during which BHA was increased from baseline was more sustained in the supragranular than in the granular and infragranular layers (73.5-, 18.7-, and 20.0-ms median supragranular, granular, infragranular, respectively; both Wilcoxon tests, $P<0.001)$. In contrast, the poststimulus time interval during which MUA was increased from baseline was shorter in the superficial, as compared to deeper layers (15.5-, 42.5-, and 45.5-ms median supragranular, granular, and infragranular, respectively; both Wilcoxon tests, $P<0.001)$. Neither BHA nor MUA durations differed between granular and infragranular layers (both Wilcoxon tests, $P>0.84$ ). While the spatiotemporal pattern of BHA and MUA differences in A1 largely parallels with that observed in V1, there are noteworthy A1-V1 differences. BHA in V1 during both passive (stimulus locked) and active (i.e., fixation locked) visual input had two elements (early and late) with confined laminar spread; the early component was limited to deep layers, while the late component was limited to supragranular layers. In $\mathrm{A} 1$, we observed larger temporal separation between early and late BHA components (median of $33 \mathrm{~ms}$ ). As in V1, the late BHA component in $\mathrm{A} 1$ is largely limited to the superficial layers. However, unlike the early-deep component in V1, the early BHA component in A1 extends up into the supragranular layers. We quantified this by directly comparing the BHA magnitude in A1 across layers in early ( 0 - to $30-\mathrm{ms}$ poststimulus) and late (31 to $100 \mathrm{~ms}$ ) time window. Differential laminar distribution was noted for the magnitude of the late (KW test, $P<0.001$ ) but not the early BHA (KW test, $P=0.76$ ). In contrast, MUA was decreased in the supragranular layers compared to granular and infragranular layers during both time windows (all Wilcoxon tests, $P<0.001$ ).

To test whether these results further generalize to other auditory stimuli, we analyzed data from the local best frequency tone response for each site within the same penetrations in A1 (A1-BFT; $n=26$ sessions, two animals). For each individual penetration, we compared MUA responses across a set of 14 pure tones from 354 to $32 \mathrm{kHz}$ (see Materials and Methods). We defined the best frequency tone as the one that elicited the largest magnitude MUA response in the layer L4 [e.g., $(21,22)]$. Subsequently, we aggregated laminar BHA and MUA responses elicited by these best tone frequencies. This analysis reproduced our initial finding of nonuniform laminar distribution of BHA and MUA (KW test, both BHA and MUA, $P<0.01$ ), with BHA being, again, strongest in the superficial layers (all Wilcoxon test, $z>3.3, P<0.01$ ) and MUA being weakest in the superficial layers (all Wilcoxon test, $z>2.9, P<0.001$; Fig. 3 , E to H).

\section{Supragranular layers are the major source of BHA at the pial surface}

Next, we evaluated the extent to which these two BHA components (deep versus superficial) contribute to the BHA recorded on the pial surface [i.e., electrocorticographic (ECoG)-like signal; Fig. 4, A to D]. 
A
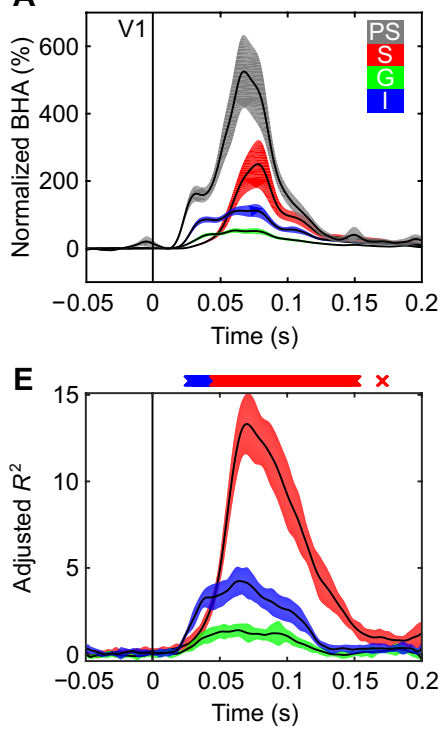

B

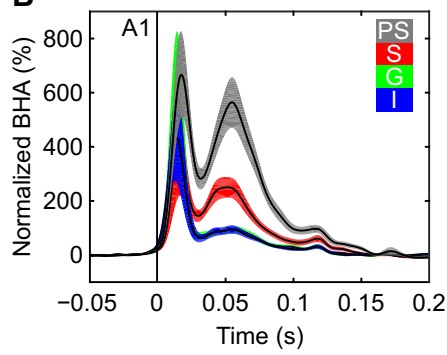

$\mathbf{F}$

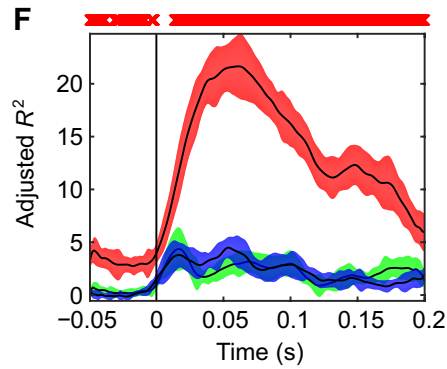

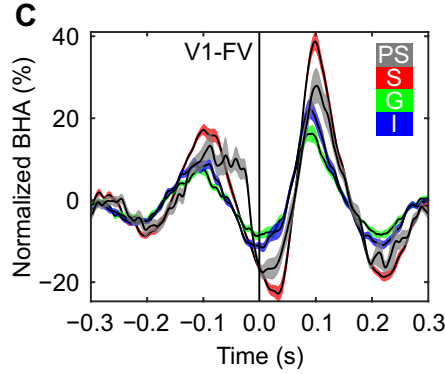

G

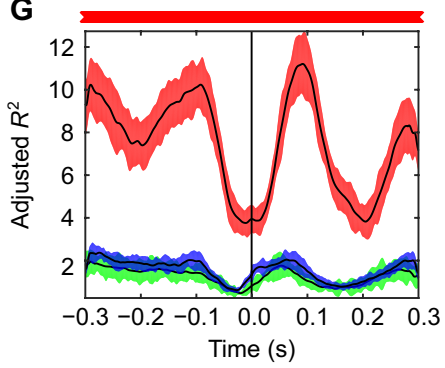

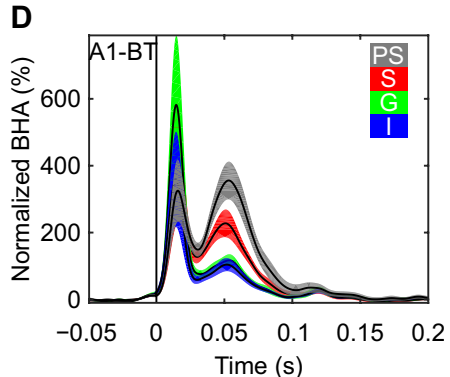

H

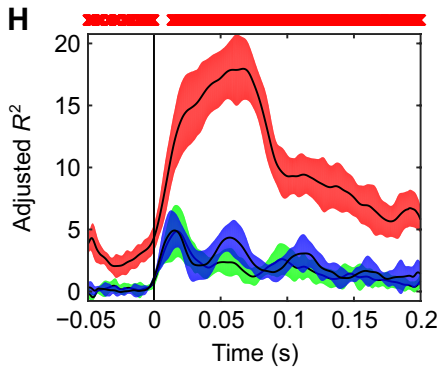

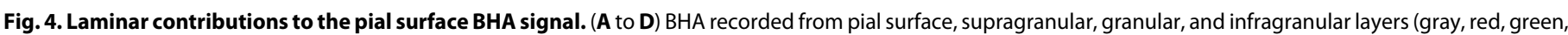

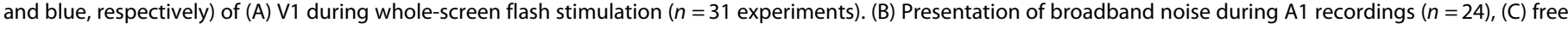

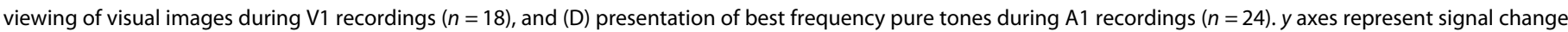

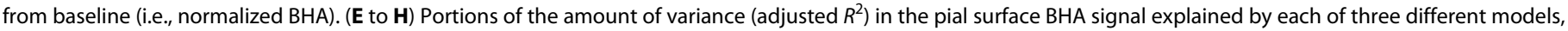

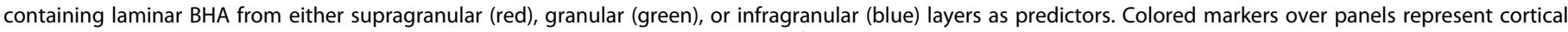
depth, which explains most of the variance (KW test across distributions of adjusted $R^{2}$ values).

We reasoned that a signal from the cortical depth that explains most of the variance in the pial surface BHA would indicate a major laminar generator of surface BHA. To quantify the contribution of generators from different depths to the pial surface BHA, we used a standard method of tracing continuous FP from a neural source (generator) to the cortical surface $[(22,23)$; see Materials and Methods]. On the basis of both the magnitude of supragranular BHA and the proximity of supragranular current generators to the pial surface of cortex, we predicted that the supragranular layers should provide the largest contribution to BHA signal at the cortical surface. To quantify this, we estimated the percentage of variance explained (adjusted $R^{2}$ ) by four linear regression models, including the data from either individual layers (Fig. 4) or all layers together (fig. S8). We used a linear regression model because it has been shown that volume conduction itself is linear at a macroscopic scale (23-25). For both V1 and A1 experiments, most of the variance was explained by supragranular BHA (see Fig. 4, E to H; KW test, $P<0.05$ ). We also observed that BHA from deep layers could explain some significant portion of variance, particularly in $\mathrm{V} 1$ passive viewing. The amount of variance explained by supragranular layers reached $15,25,12$, and $20 \%$ (for V1-DF, V1-FV, A1-BBN, and A1-BFT, respectively), while it peaked at about $5 \%$ for granular and infragranular layers in all four experiments. Thus, while the BHA from all laminar compartments contributes to the pial surface BHA, the supragranular layers appear to be the major source.

\section{Do these findings in macaques relate to BHA signals measured in humans?}

To test whether the spatial dissociation between BHA and MUA noted in monkeys could be observed in humans, we used a small dataset from similar laminar probes implanted in a to-be-resected tissue of the prefrontal cortex (PFC) of two patients with pharmacoresistant epilepsy during rest (Fig. 5). In short, spatial distributions of BHA and MUA were notably similar to those observed in monkeys with strongest BHA in the superficial electrodes and MUA in the deeper electrodes. As in our monkey data, we found nonuniform distributions across cortical depth for both BHA and MUA (both KW tests, $P<0.001)$. There was also a differential distribution of BHA and MUA across cortical depth that was similar to that observed in monkeys: BHA (Fig. 5, A and B) was strongest in the superficial channels as compared to middle and deep channels (both Wilcoxon tests, $P<0.001$ ), whereas MUA (Fig. 5, C and D) was strongest in the middle channels as compared to superficial and deep (both Wilcoxon tests, $P<0.001$ ). This is consistent with our results from nonhuman primates, showing that the main generators of $\mathrm{BHA}$ are localized in supragranular layers, whereas the strongest local population neuronal firing is observed in deeper layers. These data were recorded from only two patients during rest ( $n=960$ and 2532 segments for a duration of $1 \mathrm{~s}$, resulting in a total of 16 and $42.20 \mathrm{~min}$ of data) and should be interpreted with caution. However, the data do suggest that BHA and MUA might dissociate in the human neocortex in a manner similar to that we observed in the nonhuman primate neocortex.

\section{BHA depends on intact NMDA transmission}

Laminar activity profile recordings in rodents suggest that the most prominent current sink in the supragranular layers, which corresponds to the location generating the late-superficial BHA in our study, reflects the $\mathrm{Ca}^{2+}$-dependent spiking in the apical dendrites of pyramidal neurons (26). The synaptically evoked $\mathrm{Ca}^{2+}$ signal is largely mediated by the $N$-methyl-D-aspartate (NMDA) receptors (27), which regulate several processes, including neural plasticity (28) and dynamic shifts in neural excitability (29). The effect of NMDA-mediated enhancement of excitability is strongest in the supragranular layers (29), consistent with the preferential expression of NMDA receptors there 

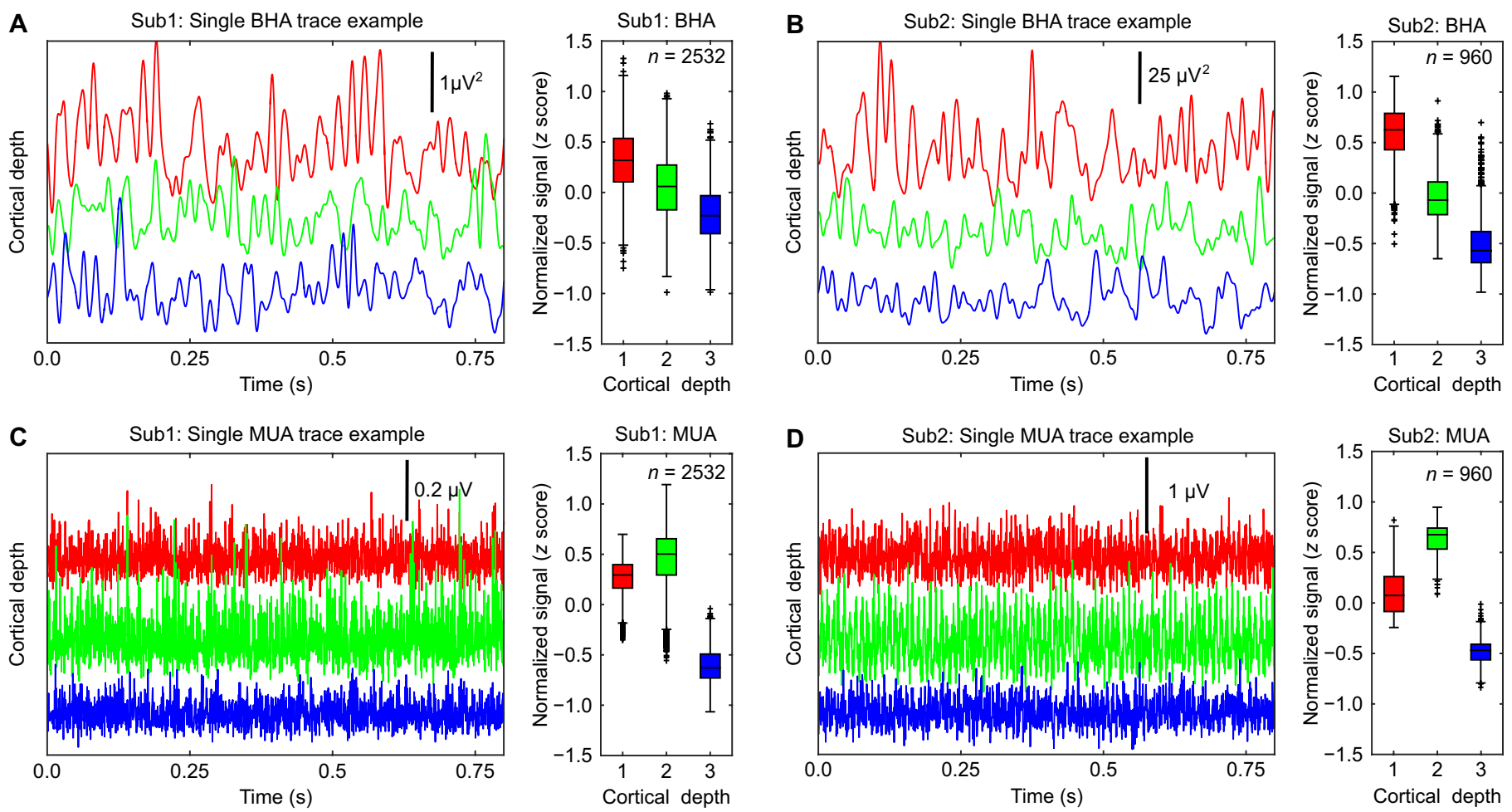

Fig. 5. Laminar distribution of BHA and MUA in the human prefrontal cortex. Line plots show example traces from simultaneous BHA (A and B) and MUA (C and D) recordings during rest in two patients with epilepsy implanted with linear probes in a to-be-resected tissue. Signals were assigned into three a priori depths (1, 150 to $900 \mu \mathrm{m} ; 2,1050$ to $1800 \mu \mathrm{m}$; and 3, 1950 to $2850 \mu \mathrm{m}$ ) based on known thickness of the cortex (see Materials and Methods). The whole resting data were segmented into 1-s-long intervals (100-ms-long intervals at side of epoch were removed after filtering to avoid contamination of edge effects). The laminar distribution of BHA and MUA was quantified by testing for nonuniformity of BHA and MUA across depths (see Materials and Methods). Box plots present results aggregated across all BHA (A and $\mathrm{B}$ ) and MUA ( $C$ and $D)$ epochs in each patient separately. Box plots indicate 25th percentile, median, and 75th percentile; whiskers extend to extreme values not considered outliers. Outliers are shown as " + " signs.

(30). The laminar distribution of NMDA receptors and the time course of the NMDA-mediated depolarization (slower and primarily supragranular) suggest that the later superficial BHA signal may have a strong NMDA dependence, further dissociating BHA from neuronal firing. To address this possibility, we examined the effects of systemic administration of the noncompetitive NMDA antagonist phencyclidine (PCP) on auditory responses in A1 ( $n=8$ experiments, one animal). During control recordings (i.e., before PCP), stimulation elicited a sharp and transient increase in both BHA and MUA (Fig. 6). To test the overall effect of PCP on BHA and MUA, we averaged each signal across time within a $150-\mathrm{ms}$ poststimulus window and compared the magnitude of these averaged responses before and after PCP administration (see Fig. 6). After PCP administration, BHA was significantly attenuated across all layers (all Wilcoxon tests, $P<0.01$ ), while MUA showed no detectable difference between control and PCP (all Wilcoxon tests, $P>0.26$ ) at any cortical depth. While there is no significant impact on the MUA, both early and late BHA components are attenuated by the PCP. It is also noteworthy that the early BHA component is attenuated, while the late component is abolished. Albeit based on data from only one subject, the effects of NMDA blockade are robust and provide a pharmacological dissociation between BHA and MUA. Previous studies found enhanced [e.g., (31)] and suppressed (32) BHA after NMDA-antagonist administration. These differential effects might be attributed to the complex influence that NMDA-antagonists have on the stimulus-elicited response (32). Lazarewicz et al. (32) found that an NMDA-antagonist (ketamine) simultaneously enhanced prestimulus and poststimulus gamma activity, while the relative response to stimulus was decreased. The current findings point to a pharmacological dissociation with low doses of an NMDA-antagonist affecting BHA relative response to stimulus while keeping MUA intact.

\section{DISCUSSION}

BHA is a critical mesoscopic signal commonly used to bridge the gap between human electrophysiology and single-unit studies in animals. Moreover, BHA is the closest neural correlate to the BOLD fMRI signal, linking neuroimaging and neurophysiology. On the basis of influential earlier studies, a prevalent view on human electrophysiology is that BHA is a simple reflection of MUA. Across experiments in $\mathrm{V} 1$ and $\mathrm{A} 1$, we identified spatial, temporal, and pharmacological dissociations between BHA and MUA. Our results suggest that BHA and MUA index different aspects of the neural activity with divergent features across layers of the neocortex. BHA had two spatially and temporally distinct components, i.e., early deep and late superficial, observed in granular-infragranular and supragranular layers, respectively. There is a substantial spatial and temporal correspondence between MUA and the early-deep BHA component. In contrast, the MUA correlate of late-superficial BHA is much weaker and often undetectable. BHA appears to be NMDA mediated, with low doses of noncompetitive NMDA antagonist (PCP) decreasing BHA while leaving MUA intact. Our regression analyses show that, although 

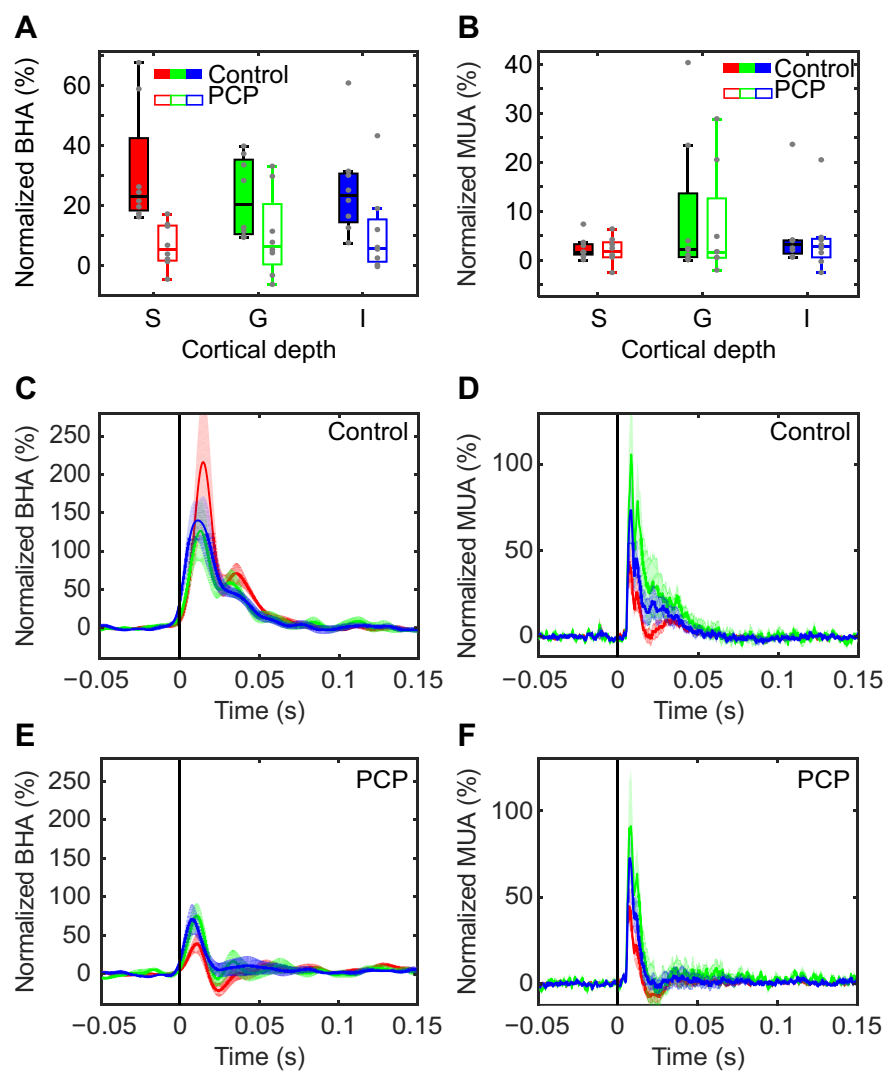

Fig. 6. The effect of PCP on BHA and MUA in the primary auditory cortex. (A and B) Box plots present BHA (A) and MUA (B) averaged within the 150-ms-long poststimulus time window before (control) and after systemic administration of PCP $(n=8)$. Box plots indicate 25th percentile, median, and 75th percentile; whiskers extend to extreme values not considered outliers. (C to $\mathbf{F}$ ) Line plots show the time course of $\mathrm{BHA}$ ( $\mathrm{C}$ and $\mathrm{E}$ ) recorded from supragranular, granular, and infragranular (red, green, and blue lines) layers of $\mathrm{A} 1$ before $(\mathrm{C})$ and after $(\mathrm{E})$ systemic administration of PCP. (D and F) Concomitant MUA recordings from the same supragranular, granular, and infragranular (red, green, and blue lines) layers before (D) and after (F) systemic administration of PCP. $x$ axis indicates time relative to stimulus onset. $y$ axis represents signal change from baseline, averaged over the 50-ms interval before the stimulus (normalized BHA/MUA). Shading reflects SEM.

the BHA from all laminar compartments volume conducts to the pial surface, the main generators of BHA are localized in the supragranular layers where, as shown by numerous prior studies, firing is sparse $[(22,33,34)$; for review, see (35)]. This is important because it suggests that the BHA recorded at the pial surface (e.g., with an ECoG electrode) likely overrepresents signals generated in supragranular layers, while the more robust event-related spiking activity is observed in the granular and infragranular layers and often precedes activation, as indexed by BHA. The results of laminar recording experiments in rodents (26) indicate that the large, late-superficial component may be generated by $\mathrm{Ca}^{2+}$ influx during apical dendritic spiking of pyramidal cells. The vulnerability of the largest (late superficial) component of BHA signal to NMDA receptor blockade in our experiments suggests that this component may index the same process.

Several critical implications of our findings for the interpretation of the BHA signal merit further emphasis. First, BHA as recorded from the pial surface of the cortex reflects relatively modest contri- butions from neuronal firing. Our pharmacological findings (Fig. 6) link to those of Suzuki and Larkum (26) in pointing to an underlying NMDA-mediated process generating BHA. One possibility is that $\mathrm{BHA}$ originates from calcium-dependent spikes that are long-lasting (10 to $100 \mathrm{~ms}$ ) nonsynaptic events triggered by NMDA receptormediated excitatory postsynaptic potentials (36) that have been suggested to be a mechanism for associating information carried by feedforward and feedback pathways (37).

The current finding that the supragranular BHA is the largest contributor to the pial surface signal suggests that the BHA as typically measured in ECoG may contain a substantial representation of input from cortical feedback pathways. The median of 33-ms (interquartile range of $3 \mathrm{~ms}$ ) onset-to-onset difference between early and late supragranular BHA signals in A1 is much longer than expected for a conduction delay within a direct monosynaptic connection between granular and supragranular layers (35). One possible explanation is that the early BHA reflects feedforward signal propagating to $\mathrm{L} 4$ and then to extragranular layers, whereas the late-superficial BHA reflects feedback from higher auditory areas to A1, which provides a strong input to the supragranular layers. In feedback pathways, predictions and contextual information originating in extragranular layers of higher-order areas are projected to and modulate lower cortical areas. Because feedforward and feedback pathways encode different information (33), bias toward feedback circuits in ECoG-derived BHA might favor predictive and contextual information.

The idea that BHA reflects an integrative process separable from the typical action potential may help to interpret several currently unexplained observations. Niessing et al. (14) used recordings from V1 of anesthetized cats to show that BHA is a better correlate of BOLD fMRI than MUA. Both BOLD and BHA encode stimulus intensity at a finer rate than MUA. This is puzzling under the assumption of BHA being a direct consequence of spiking neurons. The current results help to understand this discrepancy by suggesting that BHA (and consequently BOLD fMRI) might reflect dendritic processes subthreshold to neural firing. This interpretation would predict that the correlation between the magnitude of BHA and firing, rather than being constant, depends on the effectiveness of a stimulus in driving the cortex. For stimuli that are more effective in eliciting action potentials, correspondence between BHA and MUA will be stronger than for stimuli that are less effective. In support of this, Nir et al. (15) showed that coupling between firing rates of individual neurons and $\mathrm{BHA}$ varies across time rather than being stable. Furthermore, they also showed that the level of spike-BHA coupling depends on the degree of firing rate correlations between neighboring neurons. Similarly, Smith et al. (38) observed changing correlation between BHA and MUA during epileptic seizure. In support of the subthreshold view, Rich and Wallis (17) found that the BHA in the orbitofrontal cortex, although correlated with firing, diverged from MUA on several dimensions, carried more information, and was more sensitive to spatiotemporal changes than neural firing. The current findings suggest that $\mathrm{BHA}$ primarily reflects a dendritic process that is separable from neural firing. Our findings also suggest that using BHA as a proxy for neural firing overestimates the onset latency and duration of firing. This outcome introduces important caveats into the interpretation of the BHA signal, but it remains clear that the signal, particularly in the deeper layers, still has a strong relationship to neuronal firing. Improved understanding of the additional neuronal contributions to BHA makes it a richer, more useful index of brain activation. While it is unlikely, it is also possible that BHA 
and MUA might be generated by two distinct mechanisms operating at the same time at the same cortical depths.

The current findings provide an additional step toward the understanding that in supragranular layers, neural codes could entail dense subthreshold synaptic inputs accompanied by sparse firing (35). It has been clear for some time that the firing in superficial layers is sparse compared to those in the deeper layers. For example, Sakata and Harris (34) studied the population activity in the rat auditory cortex during spontaneous and sensory-evoked stimulation. Both conditions exhibited a sparse, spatially localized activity in the layer $2 / 3$ pyramidal cells, with a densely distributed activity in the larger layer 5 pyramidal cells and putative interneurons. Recordings from pyramidal neurons in primary somatosensory barrel cortex, both under anesthesia and in awake animals, also revealed low spontaneous and evoked firing in layer $2 / 3$ compared to much higher firing rates in layer 5 pyramidal neurons $(39,40)$. Another study that looked at the barrel cortex in awake head-restrained mice during object localization found superficial layers (layer 2/3) firing was about 20 times weaker than firing rates in deeper layers (41). Similarly, in A1 of nonhuman primates, neuronal firing is typically reduced in the superficial, relative to the middle and deep layers during both spontaneous and stimulusevoked recordings [e.g., $(21,42)$ ]. A relative paucity of firing in superficial layers is observed in primary somatosensory cortex during stimulation (43) and in resting spontaneous activity (44). Similarly, Bastos et al. (45) observed a weaker superficial firing, as compared to layer 4 in macaque prefrontal cortex (PFC) (see the Supplementary Materials). Self et al. (46) also show weaker supragranular MUA in response to full-screen, high-contrast checkerboard stimulation and during spontaneous recordings in V1. A detailed review of possible mechanisms leading to sparser firing in the superficial layers relative to deeper layers is beyond the scope of our manuscript. However, the fact that layer $2 / 3$ pyramidal neurons appear to require substantially more excitatory synaptic input to drive them to action potential threshold compared to L5 pyramids (35) points to one mechanistic explanation for this common observation.

\section{MATERIALS AND METHODS \\ Experimental design \\ Nonhuman primates data acquisition}

Data from four nonhuman primates (Macaca mulatta, 5.0 to $7.0 \mathrm{~kg}$ ) were included in the current study: two female $(n=104$ penetrations into V1 during whole-screen flashes; $n=49$ penetrations into V1 during free viewing of colored images) and two male monkeys ( $n=26$ penetrations into A1 during broadband noise presentation and best tone response). We also used data recorded from A1 in one additional male monkey ( $n=8$ penetrations) before and after systemic administration of PCP. Animals sat in a primate chair in electrically shielded and sound-attenuated chambers, with their head fixed in position. Laminar profiles of FPs and concomitant MUA were obtained using linear array multicontact electrodes, with 23 sites equally spaced at 100 or $200 \mu \mathrm{m}$. The electrode was inserted through the dura into the brain and positioned to span all layers of the cortex (Fig. 1). The impedance ranged from 0.3 to 0.5 megohms. We used a standard measure to estimate the magnitude of the spiking activity in the local neuron population surrounding each electrode contact, MUA $(47,48)$. Signals were impedance matched with a preamplifier (10× gain, bandpass DC of $10 \mathrm{kHz}$ ) situated on the electrode, and after further amplification $(500 \times)$, the signal was split into FP (0.1 to
$500 \mathrm{~Hz}$ ) and MUA (300 to $5000 \mathrm{~Hz}$ ) range by analog filtering. FPs were sampled at 2-kHz/16-bit precision, and MUA was sampled at $20-\mathrm{kHz} /$ 12-bit precision. Additional zero phase shift digital filtering (300 to $5000 \mathrm{~Hz}$ ) and rectification were applied to the MUA data, and, last, it was integrated down to $1 \mathrm{kHz}$ (sampled at $2 \mathrm{kHz}$ ) to extract the continuous estimate of cell firing $(21,22,47)$. The BHA was calculated by filtering FPs within a range of 70 to $150 \mathrm{~Hz}$ (see below for more details).

\section{Surgery}

The surgical procedures for nonhuman primates followed those previously described $(22,33)$. Briefly, preparation for awake intracranial recordings was performed using aseptic techniques under general anesthesia. The tissue overlying the calvarium was resected, and appropriate portions of the cranium were removed while overlying dura and neocortex were left intact. Custom recording chambers (Crist Instrument Co. Inc.) with electrode guide grids were positioned so that the electrode track was perpendicular to the surface of V1 or to the lower bank of the lateral sulcus for A1 penetrations; for the details of the insertion of laminar multielectrode array, see the Supplementary Materials.

\section{Visual stimuli}

Visual stimuli, consisting of both whole-screen and diffused light flashes, were used to elicit visual-evoked response profiles for the positioning of laminar electrodes to straddle the layers of V1 (49). Stimuli ( $n=100$ to 300 per penetration) were presented at a distance of $86 \mathrm{~cm}$ in front of the monkeys. Stimuli were delivered either every $642 \mathrm{~ms}$ as a whole-screen luminance flux on a video monitor (17-ms duration each) or were generated by a Grass PS33 Plus photic stimulator (Grass-Telefactor Inc., West Warwick, RI) and projected onto a diffuser at a $2-\mathrm{Hz}$ rate with a $10-\mathrm{ms}$ duration. To test whether BHA-MUA dissociation generalizes to other conditions and stimuli sets, we examined the experimental data collected during the free viewing of colorful images in the same V1 penetrations [see (20) for more details on recordings]. It is noteworthy that natural scene viewing produces a much more effective activation of the superficial layers of the primary visual cortex (20).

\section{Auditory stimuli}

Auditory stimuli consisted of broadband noise delivered at $60-\mathrm{dB}$ sound pressure level (SPL) through two free field speakers directed toward the ears (Tucker-Davis Technologies). Noise stimuli ( $n=100$ per penetration) were presented for the duration of $100 \mathrm{~ms}$ each and were interleaved with pure tones of different frequencies ranging from $354 \mathrm{~Hz}$ to $32 \mathrm{kHz}$ presented at $625-\mathrm{ms}$ stimulus onset asynchrony. The auditory stimuli used in the pharmacology experiment consisted of 100 clicks presented at 65-dB SPL with the interstimulus interval of $450 \mathrm{~ms}$. All protocols were approved by the Institutional Animal Care and Use Committee of the Nathan Kline Institute. We selected these nonoptimal stimuli (diffused flash light for V1 and broadband noise for A1) because we expected the dissociation to be strongest under these conditions, where activation of the superficial layers is relatively weak. Analysis of responses to stimuli that are not experimentally optimized for the neurons under study is not an unreasonable approach since, in the vast majority of human ECoG studies, stimuli cannot be optimized for all recording sites. The broadband noise stimulus was overall very effective in activating A1 across all layers. To further test whether the dissociation between BHA and MUA generalizes to the optimal auditory stimuli, for each penetration, we establish the best frequency of the recording site using a "suprathreshold" method [e.g., (21)]. Briefly, we examined a set of pure tones ( $354 \mathrm{~Hz}$ to $32 \mathrm{kHz}$ in half-octave steps), each lasting for $100 \mathrm{~ms}$. 
For each penetration, we identified the tone that elicited the largest MUA in the granular layer. These best frequency tones were then used in further analyses of the BHA and MUA laminar distribution.

\section{Monkey laminar assignment}

We assigned channels to layers using standard criteria described previously in V1 and A1 $(22,33,49)$. Briefly, V1 during visual stimulation exhibits a "feedforward" laminar profile, with activation beginning in layer 4 and followed by that in the extragranular layers. Therefore, the granular layer was assigned to channels showing the earliest current sink coupled with a concomitant increase in $\operatorname{MUA}(22,33,49)$. On the basis of the laminar current sink and source patterns and known anatomy (50), the positions of the supragranular and infragranular layers were identified relative to the position of granular layer. In the primary auditory cortex, the polarity of P30 in FPs inverts to negative in supragranular layers and that inversion coincides with supragranular sink in the CSD profile. Before P30, there is an early negative peak, which inverts to positive at a depth corresponding to a granular current sink that is often paired with a deeper source. The sink/source configuration in the granular layer coupled with rapid increase in MUA reflects the initial thalamocortical activation of A1 (51). For a similar approach, see $(21-23,31,33,49,51-54)$. Temporal response profiles in $\mathrm{V} 1$ and $\mathrm{A} 1$ are different. In particular, the initial input to A1 is very rapid below 10 -ms poststimulus onset $(21,51)$, while input to V1 is much slower on the order of $\sim 30$ to $50 \mathrm{~ms}$ in the very fastest cases reported $(49,55)$ and, similarly, the delay between stimulus-evoked neural firing of $\sim 30 \mathrm{~ms}$. The temporal lag in response onsets between the granular and supragranular layers is significant in V1 [e.g., $(49,55)$ ], while that in A1 is substantially smaller [e.g., (51)]. The subtle differences between stimulus-evoked MUA onset latency in layer 4 versus layers $2 / 3$ of $A 1$ are obscured by the bandpass filtering used to extract the $\mathrm{BHA}$ signal. This is also the main reason why we do not compare onsets between BHA and MUA in the current manuscript.

\section{Human data acquisition}

We also used data from two human patients with epilepsy implanted with intracranial electrodes for diagnostic purposes; the laminar probes were placed into cortical areas that were planned for later surgical excision. One subject had a laminar probe at the border between Brodmann areas 6, 8, and 9, whereas the second subject was implanted in left frontal Brodmann area 46. Both subjects 1 and 2 were males recorded during rest for the duration of 43 and $16 \mathrm{~min}$, respectively. Laminar profiles of FPs and concomitant MUA were obtained using linear array multicontact electrodes, with 23 sites equally spaced at $150 \mu \mathrm{m}$. All protocols were approved by the local Institutional Review Board at the Hungarian Academy of Sciences.

\section{Human depth assignment and analysis of the human data}

Unlike in V1 and A1, the laminar physiology of cortical areas that were implanted in human subjects (i.e., frontal cortex) remains poorly understood. We therefore assigned signals to cortical depths on the basis of the known cortical thickness of the frontal cortex, rather than anatomical and physiological markers, as described above. The thickness of the human prefrontal cortex is, on average, below $2800 \mu \mathrm{m}$ (56). Because the intercontact spacing of the laminar probes was $150 \mu \mathrm{m}$ and the silicon pad at the top of the probe rested on the cortical surface, we a priori assigned channels into three equally spaced depth bins: superficial (150 to $900 \mu \mathrm{m})$, middle (1050 to $1800 \mu \mathrm{m})$, and deep (1950 to $2850 \mu \mathrm{m})$. We therefore refer to the cortical depths rather than cortical layers in our data from human participants (Fig. 5).
The BHA and concomitant MUA in human subjects were both calculated in the same way as for the nonhuman subjects (see above), with the same filtering and artifact removal procedures. Preprocessed data were segmented into 1-s time intervals, resulting in 2532 and 960 epochs for subjects 1 and 2, respectively. These were then $z$-scored across channels and averaged within each depth bin. We used the same statistics approach to quantify the laminar distribution of the BHA and MUA as in the analysis of nonhuman primate (described above). Briefly, we first calculated KW separately for BHA and MUA to test for any difference in the laminar distributions across layers. Subsequently, we used the Wilcoxon signed-rank test to calculate pairwise comparisons between different depth bins separately for the BHA and MUA.

\section{Pharmacology study}

To test the role of $\mathrm{Ca}^{2+}$ in $\mathrm{BHA}$, we studied the activity recorded in A1 ( $n=8$ penetrations, one monkey). The animal was presented with trains of clicks (interstimulus interval of $450 \mathrm{~ms}$ ) before and after systemic administration of PCP $(0.5$ and $1 \mathrm{mg} / \mathrm{kg}, n=5$ and 3 , respectively), a noncompetitive NMDA antagonist whose effect is to block $\mathrm{Ca}^{2+}$ channels. We reasoned that if $\mathrm{BHA}$ depends on $\mathrm{Ca}^{2+}$ dynamics, then PCP would primarily influence BHA rather than MUA. All analyses were performed, as described above.

\section{Statistical analysis \\ Data preprocessing}

The FPs and MUA were first segmented relative to the stimulus (nonhuman primate data with presentation of diffused light flashes, broadband noise, and best tone response). Subsequently, BHA was calculated from FPs by filtering and Hilbert-transforming segmented data. Complex-valued time series was rectified and squared to extract power. To avoid contamination from edge effects of filtering, we discarded data from the first and last 150-ms window, resulting in $450 \mathrm{~ms}$ epochs (-150 $\mathrm{ms}$ to $300 \mathrm{~ms}$ relative to stimulus onset).

To analyze free-viewing data, we used a different approach. During presentation of 40 colored images, we identified onsets of fixations and saccades [1000 Hz; EyeLink 1000; SR Research Ltd., Mississauga, Canada; five-point calibration; for more details, see (20)] using SR Research software. Subsequently, BHA was calculated on continuous data. Next, using eye-movement events (fixation and saccade onsets), we segmented the data relative to eye event onset with $350 \mathrm{~ms}$ before and after the onset. Note that because fixation- and saccade-locked analyses revealed similar effects, we focused on fixation-locked analyses in the main manuscript. Human data were segmented into 1-s-long consecutive epochs $(100 \mathrm{~ms}$ from each side was removed to avoid edge effects of filtering).

All the above analyses used the same segmentation for the FPs and MUA signals. For laminar segmentation, we calculated one-dimensional CSD profile from FPs using the second spatial derivative. All the analyses in the main manuscript used BHA calculated on the basis of bipolar derivative (but see fig. S1). Segmented FPs were next notch filtered to remove line noise at $60 \mathrm{~Hz}$ and harmonics at 120 and $180 \mathrm{~Hz}$. Trials with excessive signal deflection in either FP or MUA exceeding $\pm 5 \mathrm{SD}$ of the mean were considered as artifacts and removed from further analyses. The same criterion was used for all the experiments presented in the manuscript.

\section{Broadband high-frequency activity}

FPs based on the first and second spatial derivative (i.e., the bipolar and CSD, respectively) have comparable spatial specificity; however, the former maximizes signal-to-noise ratio. This is because the 
calculation of the bipolar signal includes subtraction between two rather than three neighboring channels. Thus, we primarily used the bipolar montage to calculate BHA. Nevertheless, it is worth to note that the BHA based on both first and second spatial derivatives shows similar laminar distributions with the strongest generators observed in the supragranular layers (see fig. S1, A and B for CSDbased BHA in V1 and A1, respectively).

The segmented FPs were filtered in the high-frequency broadband range (70 to $150 \mathrm{~Hz}$ ) typically used as a proxy for local neural firing. The single-trial data were then Hilbert transformed, and the power time series was extracted. In a set of control analyses, neighboring frequency ranges (i.e., 70 to $170 \mathrm{~Hz}$ and 70 to $200 \mathrm{~Hz}$; figs. S2 and S3) were explored to ensure that the laminar distribution of BHA is robust across other possible frequency windows within the BHA range. Furthermore, as expected, filtering FP at frequencies higher than $\mathrm{BHA}$ range (i.e., high-pass at $300 \mathrm{~Hz}$ ), we observed the laminar distribution that followed that of MUA (see fig. S4).

To generate the plots in Fig. 1 (E and F), we quantified the effect elicited by stimuli on the BHA and MUA by using nonparametric Wilcoxon signed-rank test. Each time point was tested relative to the prestimulus baseline interval (averaged BHA and MUA within the time window from -60 to $-10 \mathrm{~ms}$ relative to stimulus onset). To control for multiple comparisons, we used the Benjamini and Yekutieli procedure for controlling the false discovery rate (57). The same statistical procedure was used for both BHA and MUA.

Data presented in Fig. 2 were generated by aggregating BHA and MUA across all experimental sessions $(n=104$ penetrations for $\mathrm{V} 1$ whole-screen flashes in Fig. 2, A to D; $n=49$ penetrations for V1 free viewing in Fig. 2, E to $\mathrm{H} ; n=26$ penetrations for $\mathrm{A} 1$ broadband noise in Fig. 3, A to D; $n=26$ penetrations for A1 best frequency tone in Fig. 3, E to H). To further quantify the laminar distribution of BHA and MUA, we averaged each signal within 0- to 200-ms poststimulus time window and used a nonparametric KW test to quantify any differences across layers. Subsequently, we used the Wilcoxon signed-rank test for planned pairwise comparisons between pairs of layers (supragranular versus granular, granular versus infragranular, and supragranular versus granular). The data were normalized relative to a baseline interval (prestimulus in case of V1-DF, A1-BBN, and A1-BFT, and the average of the entire epoch in case of V1-FV).

\section{Cross-correlation}

Figure 1 ( $\mathrm{B}$ and $\mathrm{D})$ was generated by calculating cross-correlation between BHA and MUA elicited by light flashes and broadband noise during penetration into $\mathrm{V} 1$ and $\mathrm{A} 1$, respectively. The cross-correlation coefficient was averaged across all penetration (left) and laminar distribution of peak cross-correlation lags (right). We used KW to test whether there is any difference in the distribution of crosscorrelation lags across layers.

\section{BHA contribution to the pial surface}

To define the contribution of BHA from different depths to pial surface signal (i.e., the signal recorded with an ECoG electrode), we used a standard method of tracing continuous FP from a neural source (generator) to the brain pial surface. Similar method has been previously used to directly document both the degree of signal fall-off over distance and the quantity of signal that remains to be transported by volume conduction, with a linear signal drop-off, over long distances [e.g., $(18,22,23,51,58)]$. Here, we analyzed the BHA recorded from electrode contacts located just above the dorsal (pial) surface of the cortex, i.e., from channels 200 to $300 \mu \mathrm{m}$ above the region of active transmembrane current flow, as defined by CSD. On the basis of known anatomy of primary sensory cortices (50), we reasoned that signal at this depth reflects FPs recorded from the pial surface of the primary sensory cortex. This situation occurred in some, but not all, of the experiments comprising our dataset (V1: $n=31$ experiments, one animal; A1: $n=24$ experiments, two animals). In case of V1, this electrode is in cerebrospinal fluid, a few hundred microns above from the pia mater and in direct electrical contact with the underlying neural tissue and its electric fields. The same approach for A1 leaves the pial electrodes at the border with the somatosensory cortical area S2. As expected from prior studies [e.g., (22)], there was little evidence of the MUA signal at the pial surface. However, in contrast to MUA, the BHA signal appears to be readily volume conducted to the pial surface (Fig. 4, A to D). To understand how deep and how superficial laminar BHA components generate the pial surface BHA, we used a least squares linear model of the responses (i.e., pial surface BHA) fit to the data (i.e., laminar BHA). We estimated the variance explained (adjusted $R^{2}$ ) by each of the four models, including either BHA from one of the cortical depths (S, G, and I for BHA from supragranular, granular, and infragranular, respectively) or all the layers together ( $y \sim 1+$ BHAI, $y \sim 1+$ BHAG, $y \sim 1+\mathrm{BHAI}$, and $y \sim 1+\mathrm{BHAS}+\mathrm{BHAG}+\mathrm{BHAI})$. The procedure was repeated separately for each time point and each experiment. We reasoned that the amount of variance explained by each of these models reflects the contribution of the laminar signals to the pial surface BHA. We used a constant term in our regression model to avoid making inferences outside of the observed range of data.

For each experimental session, we fitted a separate regression across trials for each time point. We log-transformed dependent variables and fitted the models on log-transformed data, as previously recommended for skewed residual distributions [e.g., (59)]. After log-transforming the data, we did not observe any strong violations of normality.

This approach was used to generate Fig. 4 and fig. S8. Because we calculated the linear regression on single time points, we have a single estimate of variance explained at each time point per individual penetration. This allows us to track the contribution of the laminar BHA on pial surface across time. To test which depth BHA explains most of pial surface signal, we compared the distributions of adjusted $R^{2}$ values using nonparametric Wilcoxon signed-rank test and KW test, both controlled for multiple comparisons across time with the Benjamini and Yekutieli procedure (57). Briefly, for each time point in the epoch, we first identified whether any of the models explain a significant portion of variance. To this end, for each of the models, we tested whether the distribution of adjusted $R^{2}$ values differed from zero (Wilcoxon test). Next, we directly compared the amount of variance explained by different models using KW test.

Note that we used adjusted $R^{2}$ rather than $R^{2}$ to account for the fact that one of our models had more predictors. Adjusted $R^{2}$ is a modified version of $R^{2}$ adjusted with the number of predictors. Penalizing for adding unnecessary features, it allows a comparison of regression models with a different number of predictors: Adjusted $R^{2}=1-\left(1-R^{2}\right) *(n-1) /(n-k-1)$, where $n$ is the number of observations, and $k$ is the number of explanatory variables.

The value of adjusted $R^{2}$ is always less than the value of $R^{2}$ and can be negative. It increases only if the new term improves the model more than expected by chance. The value of adjusted $R^{2}$ decreases when a predictor improves the model by less than expected by chance. 


\section{SUPPLEMENTARY MATERIALS}

Supplementary material for this article is available at http://advances.sciencemag.org/cgi/ content/full/6/33/eabb0977/DC1

View/request a protocol for this paper from Bio-protocol.

\section{REFERENCES AND NOTES}

1. N. Mesgarani, E. F. Chang, Selective cortical representation of attended speaker in multi-talker speech perception. Nature 85, 233-236 (2012).

2. C. Tang, L. S. Hamilton, E. F. Chang, Intonational speech prosody encoding in the human auditory cortex. Science 357, 797-801 (2017).

3. J. Jacobs, M. J. Kahana, Neural representations of individual stimuli in humans revealed by gamma-band electrocorticographic activity. J. Neurosci. 29, 10203-10214 (2009).

4. T. Golan, I. Davidesco, M. Meshulam, D. M. Groppe, P. Mégevand, E. M. Yeagle, M. S. Goldfinger, M. Harel, L. Melloni, C. E. Schroeder, L. Y. Deouell, A. D. Mehta, R. Malach, Increasing suppression of saccade-related transients along the human visual hierarchy. elife 6, e27819 (2017).

5. M. Leszczynski, T. Staudigl, L. Chaieb, S. J. Enkirch, J. Fell, C. E. Schroeder, Saccadic modulation of neural activity in the human anterior thalamus during visual active sensing. bioRxiv 2020.03.30.015628 [Preprint]. 31 March 2020. https://doi. org/10.1101/2020.03.30.015628.

6. A. Flinker, A. Korzeniewska, A. Y. Shestyuk, P. J. Franaszczuk, N. F. Dronkers, R. T. Knight, N. E. Crone, Redefining the role of Broca's area in speech. Proc. Natl. Acad. Sci. U.S.A. 112, 2871-2875 (2015).

7. J.-P. Lachaux, N. Axmacher, F. Mormann, E. Halgren, N. E. Crone, High-frequency neural activity and human cognition: Past, present and possible future of intracranial EEG research. Prog. Neurobiol. 98, 279-301 (2012).

8. M. T. Kucewicz, J. Cimbalnik, J. Y. Matsumoto, B. H. Brinkmann, M. R. Bower, V. Vasoli, V. Sulc, F. Meyer, W. R. Marsh, S. M. Stead, G. A. Worrell, High frequency oscillations are associated with cognitive processing in human recognition memory. Brain 137, 2231-2244 (2014).

9. M. Leszczyński, J. Fell, N. Axmacher, Rhythmic working memory activation in the human hippocampus. Cell Rep. 13, 1272-1282 (2015).

10. M. Leszczynski, J. Fell, O. Jensen, N. Axmacher, Alpha activity in the ventral and dorsal visual stream controls information flow during working memory. bioRxiv 180166 [Preprint]. 24 August 2017. https://doi.org/10.1101/180166.

11. B. Voytek, A. S. Kayser, D. Badre, D. Fegen, E. F. Chang, N. E. Crone, J. Parvizi, R. T. Knight, M. D'Esposito, Oscillatory dynamics coordinating human frontal networks in support of goal maintenance. Nat. Neurosci. 18, 1318-1324 (2015).

12. M. Haller, J. Case, N. E. Crone, E. F. Chang, D. King-Stephens, K. D. Laxer, P. B. Weber, J. Parvizi, R. T. Knight, A. Y. Shestyuk, Persistent neuronal activity in the human prefrontal cortex links perception and action. Nat. Hum. Behav. 2, 80-91 (2018).

13. R. Mukamel, H. Gelbard, A. Arieli, U. Hasson, I. Fried, R. Malach, Coupling between neuronal firing, field potentials, and FMRI in the human auditory cortex. Science 309, 951-954 (2005).

14. J. Niessing, B. Ebisch, K. E. Schmidt, M. Niessing, W. Singer, R. A. W. Galuske, Hemodynamic signals correlate tightly with synchronized gamma oscillations. Science 309, 948-951 (2005).

15. Y. Nir, L. Fisch, R. Mukamel, H. Gelbard-Sagiv, A. Arieli, I. Fried, R. Malach, Coupling between neuronal firing rate, gamma LFP, and BOLD fMRI is related to interneuronal correlations. Curr. Biol. 17, 1275-1285 (2007).

16. S. Ray, N. E. Crone, E. Niebur, P. J. Franaszczuk, S. S. Hsiao, Neural correlates of highgamma oscillations $(60-200 \mathrm{~Hz})$ in macaque local field potentials and their potential implications in electrocorticography. J. Neurosci. 28, 11526-11536 (2008).

17. E. L. Rich, J. D. Wallis, Spatiotemporal dynamics of information encoding revealed in orbitofrontal high-gamma. Nat. Commun. 8, 1139 (2017).

18. C. E. Schroeder, C. E. Tenke, S. J. Givre, J. C. Arezzo, H. G. Vaughan Jr., Striate cortical contribution to the surface-recorded pattern-reversal VEP in the alert monkey. Vision Res. 31, 1143-1157 (1991)

19. D. H. Hubel, T. N. Wiesel, Receptive fields, binocular interaction and functional architecture in the cat's visual cortex. J. Physiol. 160, 106-154 (1962).

20. A. Barczak, S. Haegens, D. A. Ross, T. McGinnis, P. Lakatos, C. E. Schroeder, Dynamic modulation of cortical excitability during visual active sensing. Cell Rep. 27, 3447-3459. e3 (2019).

21. P. Lakatos, A. S. Shah, K. H. Knuth, I. Ulbert, G. Karmos, C. E. Schroeder, An oscillatory hierarchy controlling neuronal excitability and stimulus processing in the auditory cortex. J. Neurophysiol. 94, 1904-1911 (2005).

22. Y. Kajikawa, C. E. Schroeder, How local is the local field potential? Neuron 72, 847-858 (2011).

23. Y. Kajikawa, C. E. Schroeder, Generation of field potentials and modulation of their dynamics through volume integration of cortical activity. J. Neurophysiol. 113, 339-351 (2015).
24. A. Opitz, A. Falchier, C.-G. Yan, E. M. Yeagle, G. S. Linn, P. Megevand, A. Thielscher, A. Ross Deborah, M. P. Milham, A. D. Mehta, C. E. Schroeder, Spatiotemporal structure of intracranial electric fields induced by transcranial electric stimulation in humans and nonhuman primates. Sci. Rep. 6, 31236 (2016).

25. B. Pesaran, M. Vinck, G. T. Einevoll, A. Sirota, P. Fries, M. Siegel, W. Truccolo, C. E. Schroeder, R. Srinivasan, Investigating large-scale brain dynamics using field potential recordings: Analysis and interpretation. Nat. Neurosci. 21, 903-919 (2018).

26. M. Suzuki, M. E. Larkum, Dendritic calcium spikes are clearly detectable at the cortical surface. Nat. Commun. 8, 276 (2017).

27. Y. Kovalchuk, J. Eilers, J. Lisman, A. Konnerth, NMDA receptor-mediated subthreshold $\mathrm{Ca}^{2+}$ signals in spines of hippocampal neurons. J. Neurosci. 20, 1791-1799 (2000).

28. R. C. Malenka, R. A. Nicoll, NMDA-receptor-dependent synaptic plasticity: Multiple forms and mechanisms. Trends Neurosci. 16, 521-527 (1993).

29. C. E. Schroeder, D. C. Javitt, M. Steinschneider, A. D. Mehta, S. J. Givre, H. G. Vaughan Jr. J. C. Arezzo, N-methyl-D-aspartate enhancement of phasic responses in primate neocortex. Exp. Brain Res. 114, 271-278 (1997).

30. G. W. Huntley, J. C. Vickers, W. Janssen, N. Brose, S. F. Heinemann, J. H. Morrison, Distribution and synaptic localization of immunocytochemically identified NMDA receptor subunit proteins in sensory-motor and visual cortices of monkey and human. J. Neurosci. 14, 3603-3619 (1994).

31. T. van Kerkoerle, M. W. Self, B. Dagnino, M.-A. Gariel-Mathis, J. Poort, C. van der Togt, P. R. Roelfsema, Alpha and gamma oscillations characterize feedback and feedforward processing in the monkey visual cortex. Proc. Natl. Acad. Sci. U.S.A. 111, 14332-14341 (2014).

32. M. T. Lazarewicz, R. S. Ehrlichman, C. R. Maxwell, M. J. Gandal, L. H. Finkel, S. J. Siegel, Ketamine modulates theta and gamma oscillations. J. Cogn. Neurosci. 22, 1452-1464 (2010).

33. P. Lakatos, G. Karmos, A. D. Mehta, I. Ulbert, C. E. Schroeder, Entrainment of neuronal oscillations as a mechanism of attentional selection. Science 320, 110-113 (2008).

34. S. Sakata, K. D. Harris, Laminar structure of spontaneous and sensory-evoked population activity in the auditory cortex. Neuron 64, 404-418 (2009).

35. C. C. H. Petersen, S. Crochet, Synaptic computation and sensory processing in neocortical layer 2/3. Neuron 78, 28-48 (2013).

36. M. Häusser, N. Spruston, G. J. Stuart, Diversity and dynamics of dendritic signaling. Science 290, 739-744 (2000)

37. M. Larkum, A cellular mechanism for cortical associations: An organizing principle for the cerebral cortex. Trends Neurosci. 36, 141-151 (2013).

38. E. H. Smith, J.-y. Liou, T. S. Davis, E. M. Merricks, S. S. Kellis, S. A. Weiss, B. Greger, P. A. House, G. M. McKhann II, R. R. Goodman, R. G. Emerson, L. M. Bateman, A. J. Trevelyan, C. A. Schevon, The ictal wavefront is the spatiotemporal source of discharges during spontaneous human seizures. Nat. Commun. 7, 11098 (2016).

39. C. P. J. de Kock, B. Sakmann, Spiking in the primary somatosensory cortex during natura whisking in awake head-restrained rats is cell-type specific. Proc. Natl. Acad. Sci. U.S.A. 106, 16446-16450 (2009).

40. C. P. J. de Kock, R. M. Bruno, H. Spors, B. Sakmann, Layer- and cell-type-specific suprathreshold stimulus representation in rat primary somatosensory cortex. J. Physiol. 581, 139-154 (2007).

41. D. H. O'Connor, S. P. Peron, D. Huber, K. Svoboda, Neural activity in the barrel cortex underlying vibrissa-based object localization in mice. Neuron 67, 1048-1061 (2010).

42. Y. I. Fishman, D. H. Reser, J. C. Arezzo, M. Steinschneider, Complex tone processing in the primary auditory cortex of the awake monkey. II. Pitch versus critical band representation. J. Acoust. Soc. Am. 108, 247-262 (2000)

43. M. L. Lipton, M. C. Liszewski, M. N. O'Connell, A. Mills, J. F. Smiley, C. A. Branch, J. R. Isler, C. E. Schroeder, Interactions within the hand representation in the primary somatosensory cortex of primates. J. Neurosci. 30, 15895-15903 (2010).

44. S. Haegens, A. Barczak, G. Musacchia, M. L. Lipton, A. D. Mehta, P. Lakatos, C. E. Schroeder, Laminar profile and physiology of the $\alpha$ rhythm in primary visual, auditory, and somatosensory regions of neocortex. J. Neurosci. 35, 14341-14352 (2015).

45. A. M. Bastos, R. Loonis, S. Kornblith, M. Lundqvist, E. K. Miller, Laminar recordings in the frontal cortex suggest distinct layers for maintenance and control of working memory. Proc. Natl. Acad. Sci. U.S.A. 115, 1117-1122 (2018).

46. M. W. Self, T. Kerkoerle, R. Goebel, P. R. Roelfsema, Benchmarking laminar fMRI: Neuronal spiking and synaptic activity during top-down and bottom-up processing in the different layers of cortex. Neuroimage 197, 806-817 (2019).

47. A. D. Legatt, J. Arezzo, H. G. Vaughan Jr., Averaged multiple unit activity as an estimate of phasic changes in local neuronal activity: Effects of volume-conducted potentials. J. Neurosci. Methods 2, 203-217 (1980).

48. N. K. Logothetis, J. Pauls, M. Augath, T. Trinath, A. Oeltermann, Neurophysiological investigation of the basis of the fMRI signal. Nature 412, 150-157 (2001).

49. C. E. Schroeder, A. D. Mehta, S. J. Givre, A spatiotemporal profile of visual system activation revealed by current source density analysis in the awake macaque. Cereb. Cortex 8, 575-592 (1998). 
50. T. A. Hackett, T. M. Preuss, J. H. Kaas, Architectonic identification of the core region in the auditory cortex of macaques, chimpanzees, and humans. J. Comp. Neurol. 441, 197-222 (2001).

51. M. Steinschneider, C. E. Tenke, C. E. Schroeder, D. C. Javitt, G. V. Simpson, J. C. Arezzo, H. G. Vaughan Jr., Cellular generators of the cortical auditory evoked potential initial components. Electroencephalogr. Clin. Neurophysiol. 84, 196-200 (1992).

52. T. van Kerkoerle, M. W. Self, P. R. Roelfsema, Layer-specificity in the effects of attention and working memory on activity in primary visual cortex. Nat. Commun. 8, 13804 (2017)

53. E. Spaak, M. Bonnefond, A. Maier, D. A. Leopold, O. Jensen, Layer-specific entrainment of gamma-band neural activity by the alpha rhythm in monkey visual cortex. Curr. Biol. 22, 2313-2318 (2012).

54. A. Maier, G. K. Adams, C. Aura, D. A. Leopold, Distinct superficial and deep laminar domains of activity in the visual cortex during rest and stimulation. Front. Syst. Neurosci. 4, 31 (2010).

55. J. H. Maunsell, J. R. Gibson, Visual response latencies in the striate cortex of the macaque monkey. J. Neurophysiol. 68, 1332-1344 (1992).

56. J. H. Maunsell, J. R. Gibson, Measuring the thickness of the human cerebral cortex from magnetic resonance images. Proc. Natl. Acad. Sci. U.S.A. 97, 11050-11055 (2000).

57. Y. Benjamini, D. Yekutieli, The control of the false discovery rate in multiple testing under dependency. Ann. Stat. 29, 1165-1188 (2001).

58. B. A. Purcell, J. D. Schall, G. F. Woodman, On the origin of event-related potentials indexing covert attentional selection during visual search: Timing of selection by macaque frontal eye field and event-related potentials during pop-out search. J. Neurophysiol. 109, 557-569 (2012).

59. K. Benoit, Linear regression models with logarithmic transformations (London School of Economics, London, 2011), 22, pp. 23-36.

60. A. L. Barth, J. F. A. Poulet, Experimental evidence for sparse firing in the neocortex. Trends Neurosci. 35, 345-355 (2012)

61. C. E. Schroeder, C. E. Tenke, J. C. Arezzo, H. G. Vaughan Jr., Timing and distribution of flash-evoked activity in the lateral geniculate nucleus of the alert monkey. Brain Res. 477, 183-195 (1989).

62. A. D. Mehta, I. Ulbert, C. E. Schroeder, Intermodal selective attention in monkeys. I: Distribution and timing of effects across visual areas. Cereb. Cortex 10, 343-358 (2000).
63. C. E. Schroeder, S. Seto, J. C. Arezzo, P. E. Garraghty, Electrophysiological evidence for overlapping dominant and latent inputs to somatosensory cortex in squirrel monkeys. J. Neurophysiol. 74, 722-732 (1995).

64. D. C. Javitt, C. E. Schroeder, M. Steinschneider, J. C. Arezzo, H. G. Vaughan Jr., Demonstration of mismatch negativity in the monkey. Electroencephalogr. Clin. Neurophysiol. 83, 87-90 (1992).

65. Y. Kajikawa, J. F. Smiley, C. E. Schroeder, Primary generators of visually evoked field potentials recorded in the macaque auditory cortex. J. Neurosci. 37, 10139-10153 (2017).

66. P. Lakatos, G. Musacchia, M. N. O'Connel, A. Y. Falchier, D. C. Javitt, C. E. Schroeder, The spectrotemporal filter mechanism of auditory selective attention. Neuron 77, 750-761 (2013).

67. S. J. Givre, C. E. Schroeder, J. C. Arezzo, Contribution of extrastriate area V4 to the surfacerecorded flash VEP in the awake macaque. Vision Res. 34, 415-428 (1994).

68. S. J. Givre, J. C. Arezzo, C. E. Schroeder, Effects of wavelength on the timing and laminar distribution of illuminance-evoked activity in macaque V1. Vis. Neurosci. 12, 229-239 (1995).

\section{Acknowledgments}

Funding: B.R.A.I.N., MH111439, EYE24776, DC015780, and MH109429. Author contributions: M.L. and C.E.S. designed the study. A.B., Y.K., A.Y.F., and I.U. collected data. M.L. performed analyses. M.L. and C.E.S. wrote the manuscript. All authors contributed to the discussion and interpretation of findings and edited the manuscript. Competing interests: The authors declare that they have no competing interests. Data and materials availability: All data needed to evaluate the conclusions in the paper are present in the paper and/or the Supplementary Materials. Additional data related to this paper may be requested to the authors.

Submitted 29 January 2020

Accepted 30 June 2020

Published 12 August 2020

$10.1126 /$ sciadv.abb0977

Citation: M. Leszczyński, A. Barczak, Y. Kajikawa, I. Ulbert, A. Y. Falchier, I. Tal, S. Haegens, L. Melloni R. T. Knight, C. E. Schroeder, Dissociation of broadband high-frequency activity and neuronal firing in the neocortex. Sci. Adv. 6, eabb0977 (2020). 


\section{ScienceAdvances}

\section{Dissociation of broadband high-frequency activity and neuronal firing in the neocortex}

Marcin Leszczynski, Annamaria Barczak, Yoshinao Kajikawa, Istvan Ulbert, Arnaud Y. Falchier, Idan Tal, Saskia Haegens, Lucia Melloni, Robert T. Knight and Charles E. Schroeder

Sci Adv 6 (33), eabb0977.

DOI: $10.1126 /$ sciadv.abb0977

ARTICLE TOOLS

SUPPLEMENTARY MATERIALS

REFERENCES

PERMISSIONS http://advances.sciencemag.org/content/6/33/eabb0977

http://advances.sciencemag.org/content/suppl/2020/08/11/6.33.eabb0977.DC1

This article cites 65 articles, 17 of which you can access for free http://advances.sciencemag.org/content/6/33/eabb0977\#BIBL

http://www.sciencemag.org/help/reprints-and-permissions

Science Advances (ISSN 2375-2548) is published by the American Association for the Advancement of Science, 1200 New York Avenue NW, Washington, DC 20005. The title Science Advances is a registered trademark of AAAS.

Copyright @ 2020 The Authors, some rights reserved; exclusive licensee American Association for the Advancement of Science. No claim to original U.S. Government Works. Distributed under a Creative Commons Attribution NonCommercial License 4.0 (CC BY-NC). 